\title{
miR-367 promotes the proliferation and invasion of non-small cell lung cancer via targeting FBXW7
}

\author{
JING XU, WEIBING WU, JUN WANG, CHENJUN HUANG, WEI WEN, FEI ZHAO, XINFENG XU, \\ XIANGLONG PAN, WEI WANG, QUAN ZHU and LIANG CHEN \\ Department of Thoracic Surgery, The First Affiliated Hospital \\ of Nanjing Medical University, Nanjing, Jiangsu 210029, P.R. China
}

Received June 27, 2016; Accepted August 6, 2016

DOI: $10.3892 /$ or.2016.5314

\begin{abstract}
The involvement of miR-367 in lung cancer development remains unclear. In the present study, we analyzed the expression of miR-367 in tumor and adjacent tissue samples from 113 patients with non-small cell lung cancer (NSCLC) utilizing real-time PCR. miR-367 expression was significantly upregulated in the cancer tissues compared with non-cancer controls. Based on the median value of the miR-367 expression level, we divided the NSCLC patients into miR-367 high-expression and miR-367 low-expression groups. Overexpression of miR-367 was correlated with a poorer prognosis of NSCLC patients Chi-square $\left(\chi^{2}\right)$ test showed a significant statistical correlation between tumor size, tumor stage, metastasis and miR-367 expression. Additionally, miR-367 expression was found to be negatively correlated with FBXW7 expression. Based on the above correlations, we performed a series of functional experiments to further confirm the effect of miR-367 on NSCLC. Our results indicated that miR-367 may be involved in the development and progression of NSCLC by promoting proliferation and invasion and impeding apoptosis in NSCLC cells. Furthermore, FBXW7 was identified as a potential target of miR-367, and FBXW7 silencing partially compromised the invasive, proliferative and migratory capacities in the cells with low miR-367 expression. Thus, the miR-367/FBXW7 axis may be involved in the development and progression of NSCLC and may be valuable as a therapeutic target for the treatment of human NSCLC, especially cancers with high invasive potential.
\end{abstract}

Correspondence to: Dr Liang Chen or Dr Quan Zhu, Department of Thoracic Surgery, The First Affiliated Hospital of Nanjing Medical University, 300 Guangzhou Road, Nanjing, Jiangsu 210029, P.R. China

E-mail: clbright0909@njmu.edu.cn

E-mail: chest2006@163.com

Key words: miR-367, proliferation, invasion, FBXW7, NSCLC

\section{Introduction}

Lung cancer is the first leading cause of cancer-related deaths worldwide $(1,2)$. More than $75 \%$ of lung cancers are non-small cell lung cancer (NSCLC) $(3,4)$, and only a handful of NSCLC patients are diagnosed at the early stage (5). To date, the main treatment for NSCLC is surgical resection, while the relapse rate after surgery is very high and stage II-III patients are treated with adjuvant chemotherapy, which has been shown to finitely improve patient survival in several randomized clinical trials $(3,6)$. Although there has been great advances in traditional treatments, such as modus operandi and supplementation with adjuvant chemotherapy, a considerable number of patients with NSCLC are diagnosed at an advanced stage resulting in poor prognosis $(7,8)$. Therefore, novel bio-targets for therapeutic approaches to prevent cancer cell invasion and metastasis are critical for NSCLC treatment $(9,10)$.

It has been reported that FBXW7 acts as a tumor suppressor in several types of cancers and targets multiple transcriptional activators and proto-oncogenes for ubiquitin-mediated degradation (11), including cyclin E, c-Myc $(12,13)$, Notch (14), c-Jun (15), p53, mammalian target of rapamycin, and MCL1 (16,17). Abnormal expression of the FBXW7 gene is found in ovarian, breast, endometrial, renal, lymphoma and colorectal cancers (18-24). Therefore, altered expression of FBXW7 is recognized as one of the major causes of carcinogenesis or cancer development. Previous studies have clarified that loss of FBXW7 is significantly associated with a poor prognosis in breast cancer, gastric cancer, and glioma. Similarly, low expression of FBXW7 was reported to be correlated with a poor prognosis in NSCLC (25). However, the specifical mechanism underlying the above correlation remains unknown.

MicroRNAs (miRNAs) are a large class of endogenous non-coding RNAs that regulate human gene expression (26). Increasing evidence suggests that miRNAs can act as oncogenes or tumor suppressors during the development and progression of cancers through sequence-specific binding to their mRNA targets (27). These miRNAs play a vital role in a wide variety of complex biological processes, including cellular development and differentiation, but investigations have only begun to verify their significance in carcinogenesis. Given the critical correlations between FBXW7 and the prognosis 
of NSCLC (28), we hypothesized that FBXW7 is regulated by miRNAs at the post-transcriptional level. In our previous study, we identified miR-367 as a regulator of FBXW7 expression in human NSCLC cells. Our findings demonstrated that miR-367 promoted the invasion and metastasis and prevented the apoptosis of NSCLC cells via direct regulation of FBXW7. In addition, we also focused on the correlation between miR-367 and prognosis of NSCLC and found that miR-367 may be a potential biomarker for predicting the survival of NSCLC patients.

\section{Materials and methods}

Clinical tissues and cell culture. We analyzed tumor specimens from 113 patients with lung cancer who underwent surgery for excision of a primary tumor at the Department of Thoracic Surgery, The First Affiliated Hospital of Nanjing Medical University (Nanjing, China). Written informed consent was obtained from the patients, in accordance with the institutional guidelines, before sample collection, and the study was approved by the Committees for the Ethical Review of Research at Nanjing Medical University. Fresh-frozen and/ or formalin-fixed and paraffin-embedded samples were used for miR-367 and FBXW7 expression analysis. The human lung cancer cell lines H226, H1792, and A549 were cultured in RPMI-1640 medium supplemented with 10\% FBS (both from Invitrogen Life Technologies, Carlsbad, CA, USA), $100 \mathrm{U} / \mathrm{ml}$ penicillin, and $100 \mu \mathrm{g} / \mathrm{ml}$ streptomycin in a $5 \% \mathrm{CO}_{2} / 95 \%$ air at $37^{\circ} \mathrm{C}$. These cell lines were obtained from the American Type Culture Collection (ATCC; Manassas, VA, USA).

Plasmids and transfection. Luciferase constructs were generated by ligating oligonucleotides containing the wild-type or mutant putative target site of the FBXW7 3'-UTR into the luciferase reporter plasmid pEZX-MT01 vector (GeneCopoeia, Inc., Rockville, MD, USA) downstream of the luciferase gene. FBXW7 CDS sequences were cloned into the pcDNA3 vector. All sequences were completely sequenced. We conducted the transfections by using a Lipofectamine 2000 kit (Invitrogen). Cell lines were transfected with miR-367 mimics or inhibitors and their controls (GenePharma, Shanghai, China). The short interfering RNAs targeting FBXW7 (Santa Cruz Biotechnology, Inc., Santa Cruz, CA, USA) were transfected into cells using Lipofectamine 2000 reagent (Invitrogen) according to the manufacturer's instructions. The transfection efficiency is $>70 \%$ (microRNA) and $60 \%$ (plasmid) (data not shown).

RNA isolation and real-time PCR. Total RNA was isolated from tissues and cells using TRIzol (Invitrogen) and small RNA enrichment was conducted using a miRVana miRNA isolation kit (Ambion, Inc., Austin, TX, USA), according to the manufacturer's instructions. Small RNAs were reverse transcribed to cDNA using the TaqMan MicroRNA reverse transcription kit (Applied Biosystems, San Diego, CA, USA) according to the manufacturer's instructions. Then cDNA was used as a template for the real-time PCR amplification. Realtime PCR was run on a StepOnePlus ${ }^{\mathrm{TM}}$ real-time PCR instruments (Applied Biosystems). The primers used are available upon request. Real-time PCR using SYBR-Green (Takara
Bio, Inc., Otsu, Japan) was performed to compare the relative expression levels of FBXW7 mRNA according to the manufacturer's instructions. For miR-367 real-time PCR, a commercial Hairpin-it ${ }^{\mathrm{TM}}$ miRNAs qPCR Quantification kit with primers was purchased from GenePharma. The primers for the mRNAs for real-time PCR are: FBXW7 forward, 5'-GGCCAAAATGA TTCCCAGCAA-3' and reverse, 5'-ACTGGAGTTCGTGAC ACTGTTA-3'.

Western blot assay. Total proteins were extracted from the cultured cells or tissues and quantified using a BCA protein assay kit (Beyotime, Jiangsu, China) with BSA as a standard. Equal amounts of protein from different cells were separated by $10 \%$ SDS-PAGE and transferred to a PVDF membrane. The membranes were blocked with $5 \% \mathrm{BSA}(5 \% \mathrm{w} / \mathrm{v}$ in $\mathrm{PBS}+0.1 \%$ Tween-20) and incubated with primary antibodies at room temperature. The antibodies against FBXW7 and GAPDH were used according to the manufacturer's instructions (Santa Cruz Biotechnology, Inc.). After using the secondary antibodies (Santa Cruz Biotechnology, Inc.) at 1:2,000 (v/v) dilutions in PBS $+0.1 \%$ Tween-20, the signals were detected by the SuperSignal West Pico Chemiluminescent Substrate kit (Pierce Biotechnology, Inc., Rockford, IL, USA) according to the manufacturer's instructions.

Luciferase assay. H226 and H1792 cells were co-transfected with the luciferase reporter plasmid pEZX-MT01 (GeneCopoeia, Inc.), and the miRNA-367 and controls. Twenty-four hours after transfection, firefly and Renilla luciferase activities were measured using a Luc-Pair ${ }^{\mathrm{TM}} \mathrm{miR}$ Luciferase Assay kit (GeneCopoeia, Inc.). Each transfection was performed in triplicate and repeated three times.

Cell proliferation and apoptosis assay. Cell proliferation was performed with Cell Counting Kit-8 (CCK-8) (Dojindo, Tokyo, Japan). According to the instructions, CCK-8 reagent was added at $0,24,48$ and $72 \mathrm{~h}$ respectively after seeding $4 \times 10^{3}$ cells/well in a 96 -well plate, and incubated at $37^{\circ} \mathrm{C}$ for $2 \mathrm{~h}$. The optical density (OD) at $450 \mathrm{~nm}$ was detected using a microplate reader (Bio-Rad Laboratories, Richmond, CA, USA). Apoptosis was evaluated using an Annexin V/PI assay kit (Miltenyi Biotec, Bergisch Gladbach, Germany) according to the manufacturer's instructions. Flow cytometry with a FACSCalibur (BD Biosciences, San Jose, CA, USA) was performed to evaluate the result.

Transwell invasion assay. Cell invasion assays were evaluated using Transwell cell plates (Corning Costar, Inc., Corning, NY, USA) and $8-\mu \mathrm{m}$ pore size Matrigel invasion chambers (BD Biosciences) according to the manufacturer's instructions (29). Cells $\left(1.0 \times 10^{4}\right)$ were seeded in serum-free medium into the upper chamber and allowed to invade towards $10 \%$ FBS in the lower chamber. After a $24-\mathrm{h}$ incubation at $37^{\circ} \mathrm{C}$ and $5 \%$ $\mathrm{CO}_{2}$, the cells invaded through the membrane and adhered to the underside of the membrane. Then the invaded and migrated cells were fixed and stained with crystal violet. The images were acquired by using NIS Elements image analysis software (Nikon, Tokyo, Japan). For the membrane images, we calculated the number of migrated cells using image analysis software Image-Pro Plus 6.0 (Media Cybernetics, Bethesda, MD, USA). 
A

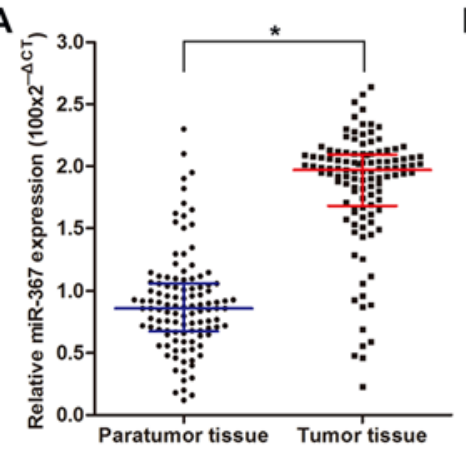

B

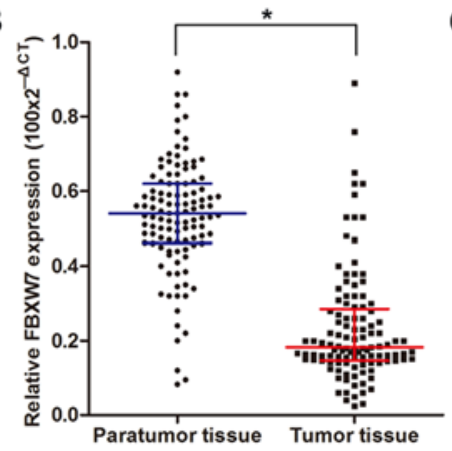

C

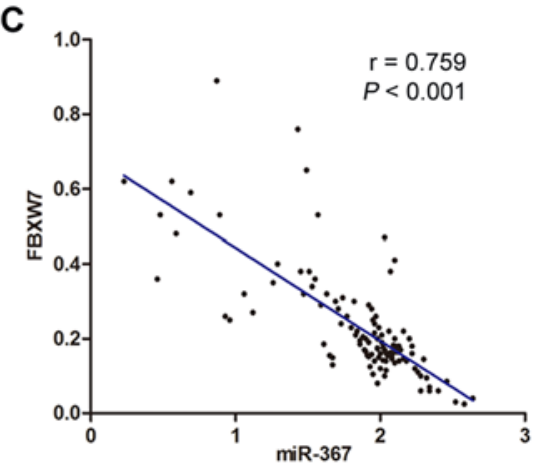

Figure 1. Expression of miR-367 and FBXW7 in NSCLC cases. Representative real-time PCR analysis of (A) miR-367 and (B) FBXW7 expression in tumor tissues and matched paratumor tissues is shown. $\mathrm{n}=113$. Values are expressed as median with interquartile range; ${ }^{*} \mathrm{P}<0.05$ by $\mathrm{t}$-test. $(\mathrm{C}) \mathrm{The}$ correlation between miR-367 and FBXW7 mRNA expression in NSCLC cases was evaluated using Spearman's correlation analysis. $\mathrm{n}=113$; $\mathrm{P}<0.001$.

Statistical analysis. All results are expressed as the mean \pm SD. The Student's t-test was used to analyze significant differences between samples. The correlation between miR-367 and FBXW7 expression levels was determined by calculating the Spearman's correlation coefficient. All the histograms were constructed using performing GraphPad Prism, version 4.0 (GraphPad Software, Inc., La Jolla, CA, USA). P<0.05 indicates a statistically significant result.

\section{Results}

Clinicopathological significance of miR-367 in NSCLC. We examined the expression level of miR-367 in 113 NSCLC clinical samples by utilizing real-time PCR, with quantified values used to calculate miR-367/U6 ratios. Our results demonstrated that the relative expression level of miR-367 was significantly higher in the cancer tissues compared with the level noted in the non-cancer tissues (Fig. 1A). Based on the median value of the miR-367 expression level, we divided the 113 NSCLC patients into two groups: miR-367 high-expression group (57 cases) and miR-367 low-expression group (56 cases). Then, we performed Chi-square $\left(\chi^{2}\right)$ tests to explore the association between the clinicopathological characteristics and miR-367 expression. The findings are summarized in Table I. There were significant differences in tumor size, tumor stage and metastatic status between the groups.

miR-367 is a negative regulator of FBXW7 in NSCLC and miR-367 modulates FBXW7 expression by directly targeting its 3 '-UTR. Increasing evidence indicates that FBXW7 is significantly associated with the prognosis of NSCLC patients. Therefore, we hypothesized that miR-367 is involved in the regulation of FBXW7. Then, we assessed the expression level of FBXW7 in the 113 NSCLC tissues via real-time PCR and found that the FBXW7 expression level in the cancer tissues was obviously lower than that in the non-cancer tissues (Fig. 1B). Pearson's correlation analysis demonstrated that the FBXW7 expression level was negatively correlated with the expression level of miR-367 in NSCLC (Fig. 1C).

To investigate the association between miR-367 and NSCLC, we transfected human NSCLC H228 and H192 cells with miR-367 mimics to upregulate the expression of miR-367, and then we assessed the expression levels of
Table I. Correlation between miR-367 expression and clinicopathological characteristics of the NSCLC patients $(n=113)$.

\begin{tabular}{lcccc}
\hline & $\begin{array}{c}\text { All } \\
\text { Characteristics }\end{array}$ & $\begin{array}{c}\text { miR-367 low } \\
\text { expression } \\
\left(<\text { median }^{\mathrm{a}}\right)\end{array}$ & $\begin{array}{c}\text { miR-367 high } \\
\text { expression } \\
\left(\geq \text { median }^{\mathrm{a}}\right)\end{array}$ & $\begin{array}{c}\text { P-value } \\
\left(\chi^{2} \text { test }\right)\end{array}$ \\
\hline $\begin{array}{l}\text { Total cases (N) } \\
\text { Age (years) }\end{array}$ & 113 & 56 & 57 & \\
$<60$ & 46 & 23 & 23 & 0.938 \\
$\geq 60$ & 67 & 33 & 34 & \\
Gender & & & & \\
Male & 59 & 28 & 31 & 0.641 \\
Female & 54 & 28 & 26 & \\
Histology & & & & \\
AC & 69 & 33 & 36 & 0.645 \\
SCC & 44 & 23 & 21 & \\
Tumor size & & & & \\
T1-T2 & 36 & 23 & 13 & $0.037^{\mathrm{b}}$ \\
T3-T4 & 77 & 33 & 44 & \\
Tumor stage & & & & \\
I-II & 39 & 25 & 14 & $0.025^{\mathrm{b}}$ \\
III-IV & 74 & 31 & 43 & \\
Metastasis & & & & \\
$\quad$ Yes & 30 & 20 & 10 & $0.029^{\mathrm{b}}$ \\
No & 83 & 36 & 47 & \\
\hline
\end{tabular}

${ }^{a}$ The median expression level of miR-367 was used as the cut-off. AC, adenocarcinoma; SCC, squamous cell carcinoma. ${ }^{b} \mathrm{P}<0.05$.

miR-367 and FBXW7 using real-time PCR. The results showed that miR-367 expression was upregulated (data not shown) while FBXW7 was significantly downregulated compared with the negative control cells. Notably, the expression of FBXW7 in the A49 NSCLC cells with downregulated miR-367 was obviously upregulated (Fig. 2A). The miRNA prediction programs, TargetScan (http://www.targetscan. org) and miRanda (http://www.microrna.org), were utilized, which predicted that a fragment of the FBXW7 3'-untranslated region (3'-UTR) contained a putative miR-367 binding 
A

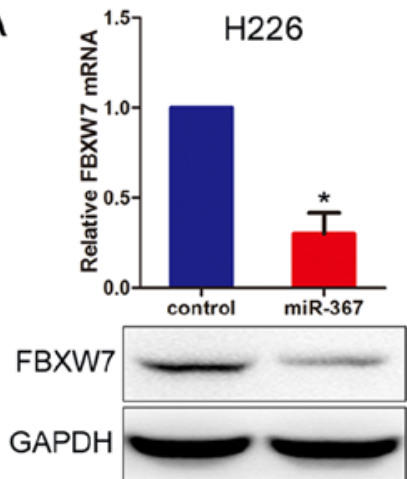

B

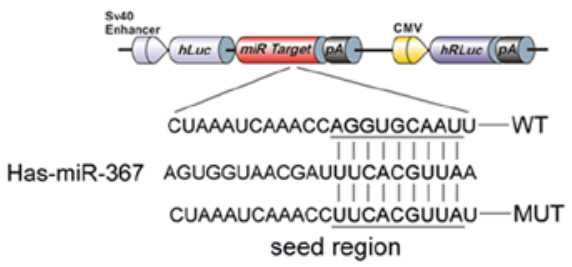

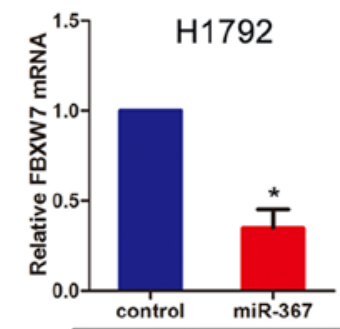

FBXW7

GAPDH

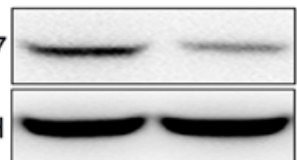

C

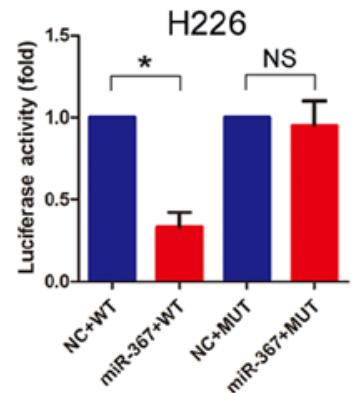

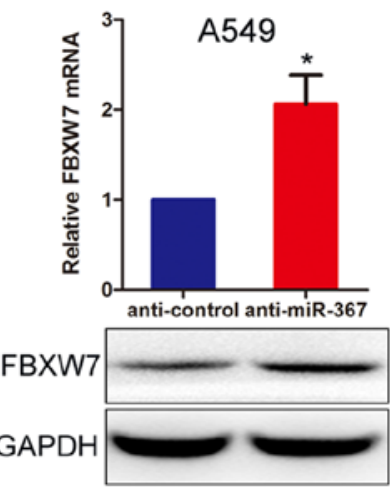

$\mathrm{H} 1792$

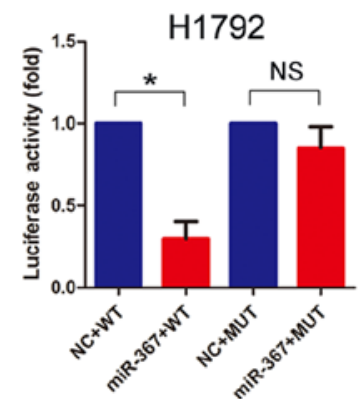

Figure 2. miR-367 downregulates FBXW7 expressions by directly targeting its 3'-UTR. (A) NSCLC cells were transfected with control or miR-367 and anti-miR-367 inhibitors. Twenty-four hours later, the expression levels of FBXW7 mRNA and protein were evaluated using real-time PCR and western blot analysis, respectively. (B) Human FBXW7 3'-UTR fragment containing the wild-type (WT) or mutant miR-367-binding sequence was cloned downstream to the luciferase reporter gene. (C) H226 and H1792 cells were co-transfected with the negative control (NC) or miR-367 mimics and the luciferase reporter construct containing the WT or mutant FBXW7 3'-UTR (MUT). For each experiment, the results were normalized to the luciferase activity detected in the cells transfected with the control vector. The results obtained from three independent experiments are presented as the mean \pm SD. NS, not significant. ${ }^{*} \mathrm{P}<0.05$ by t-test.

A

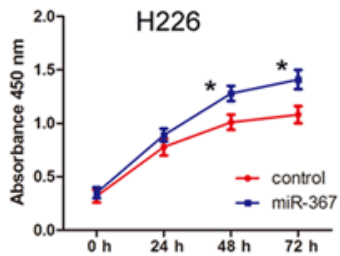

B

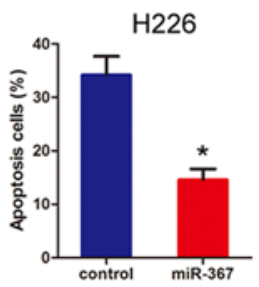

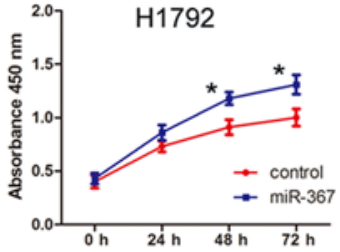

C

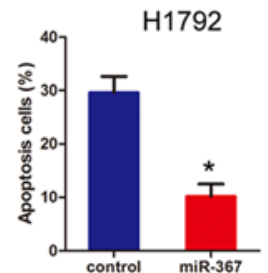

\section{C}
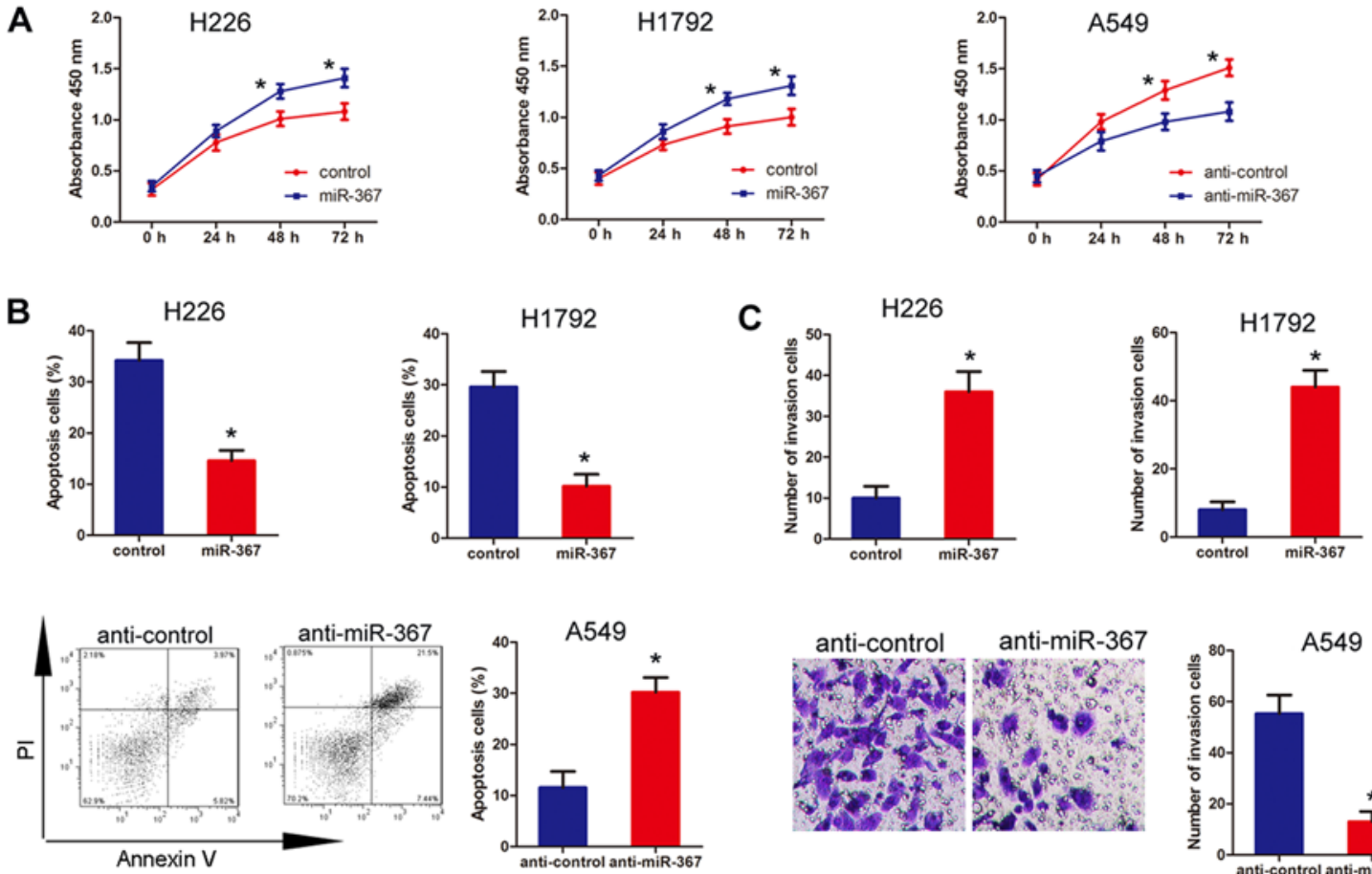

Figure 3. miR-367 promotes proliferation and invasion and inhibits apoptosis in NSCLC cells. (A) Proliferation assays were performed in the NSCLC H226 and H1792 cells transfected with the miR-367 mimics, the A549 cells transfected with the anti-miR-367 inhibitor and the control cells. Data are presented as mean \pm SD. (B) Apoptosis assays were performed using in the NSCLC H226 and H1792 cells transfected with the miR-367 mimics, the A549 cells transfected with the anti-miR-367 inhibitor and the control cells. Flow cytometry was performed to analysis cell apoptosis. (C) Invasion assays were performed in the NSCLC H226 and H1792 cells transfected with the miR-367 mimics, the A549 cells transfected with the anti-miR-367 inhibitor and the control cells. The number of cells that passed through the membrane was counted in eight fields. The results obtained from three independent experiments are presented as the mean \pm SD. ${ }^{*} \mathrm{P}<0.05$ by t-test. 


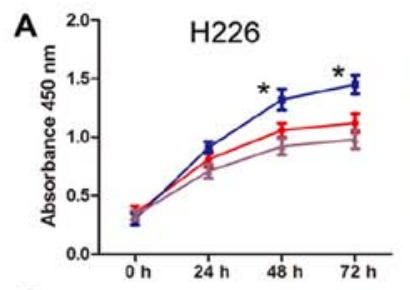

$\mathbf{B}$
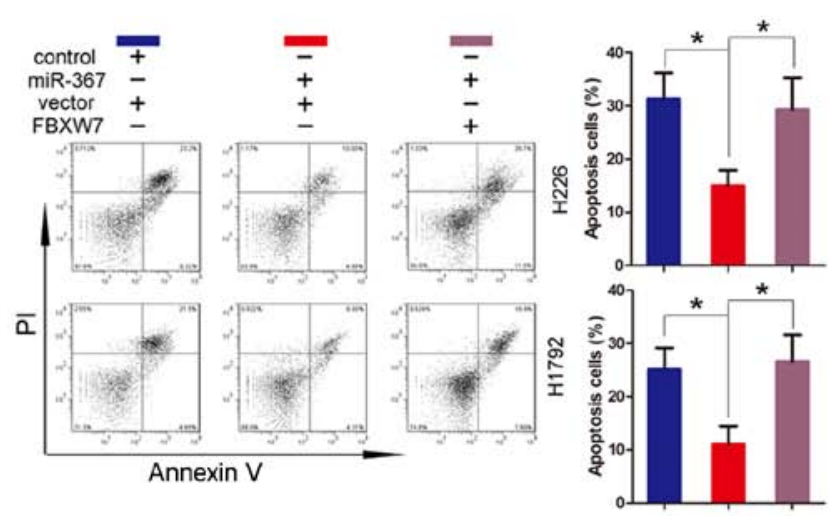

C
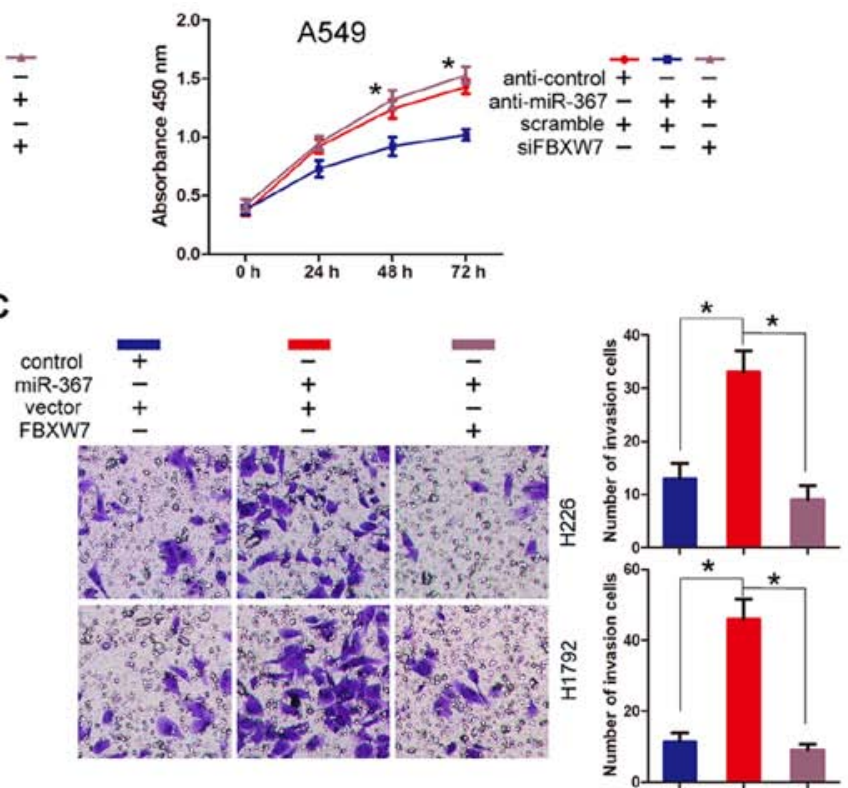
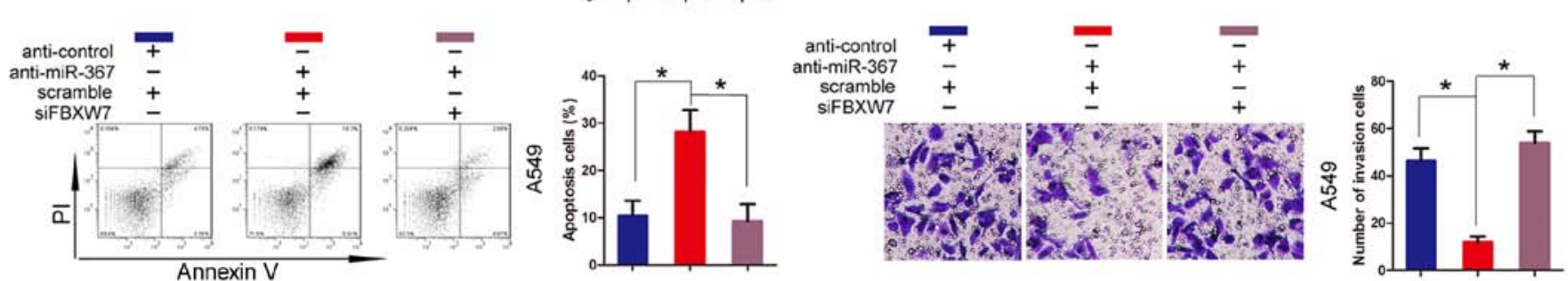

Figure 4. FBXW7 mediates the carcinogenesis-promoting function of miR-367 in NSCLC cells. (A) Proliferation assays were performed using NSCLC cells co-transfected with miR-367 mimics and FBXW7-expressing vector, or co-transfected with anti-miR-367 inhibitors and FBXW7 siRNA (siFBXW7). (B) Flow cytometric assay was performed to analyze cell apoptosis. (C) Invasion assays were performed in the NSCLC cells. The number of cells that passed through the membrane was counted in eight fields. The results obtained from three independent experiments are presented as the mean $\pm \mathrm{SD}$. ${ }^{*} \mathrm{P}<0.05$ by $\mathrm{t}$-test.

site. To further confirm the prediction, miR-367 mimics were co-transfected with a luciferase reporter construct containing wild-type and mutant FBXW7 3'-UTR (Fig. 2B). As shown in Fig. 2C, we co-transfected the miR-367 mimics with the luciferase reporter construct containing wild-type or mutant FBXW7 3'-UTR, and the cells transfected with the miR-367 mimics had downregulated luciferase activity compared with transfection with the control. Together, the results indicated that miR-367 is a potential regulator of FBXW7.

miR-367 promotes proliferation and invasion and inhibits apoptosis in NSCLC cells. The significant correlation between miR-367 expression and clinicopathological characteristics of the NSCLC cases suggested that miR-367 may play a vital role in the development and progression of NSCLC. Based on the expression pattern of miR-367, the influence of the overexpression of miR-367 on cell proliferation, apoptosis, and invasion was examined in human NSCLC cell lines H229 and H1792. The cell lines were transfected with miR-367 mimics and the expression of miR-367 was confirmed by real-time PCR (data not shown). The results demonstrated that upregulation of miR-367 promoted the proliferation, invasion and prevented the apoptosis ability compared with the negative control in NSCLC cells (Fig. 3). Moreover, to further investigate the effects of miR-367 in vitro, we transfected NSCLC A549 cells with miR-367 inhibitors to downregulate miR-367 expression.
Results of the loss-of-function experiments indicated that downregulation of miR-367 prevented proliferation, invasion and induced apoptosis compared with the negative control (Fig. 3). Taken together, our findings verified that miR-367 could promoted proliferation, invasion and inhibit apoptosis in NSCLC cells.

FBXW7 mediates the function of miR-367 in NSCLC cells. Given the correlation between miR-367 and FBXW7, we performed a rescue experiment to further examine whether the functional effects of miR-367 on NSCLC cell lines was exerted via targeting FBXW7. According to our previous results, FBXW7 was downregulated in the NSCLC cells which were then transfected with miR-367 mimics. Therefore, we co-transfected NSCLC cell lines, H226 and H1792, with pcDNA3-FBXW7 and miR-367 mimics. The transfection cells transfected with the miR-367 mimics and the co-transfected cells with the vector control and miR-367 mimics were used as the control groups. The proliferation assay showed that overexpression of FBXW7 partially abolished the promotion of cell proliferation induced by miR-367 (Fig. 4A). Then, we performed an apoptosis assay. The results indicated that apoptosis partially increased when H226 and H1792 cells were co-transfected with pcDNA3-FBXW7 and miR-367 mimics (Fig. 4B). Similarly, invasive ability was reversed to some extent when FBXW7 was overexpressed in the cells with 
high-expression of miR-367 compared to the control (Fig. 4C). Moverover, we co-transfected human NSCLC cell line A549 with siRNA-FBXW7 and miR-367 inhibitors. Then, we performed the proliferation, apoptosis, and Transwell invasion assay. Notably, the findings indicated that proliferation, apoptosis, and invasion were reversed to some extent when we silenced FBXW7 in cells with low expression of miR-367 compared to the control (Fig. 4). In conclusion, these results indicated that the effect of miR-367 on NSCLC cell lines was partially dependent on FBXW7.

\section{Discussion}

Our present study demonstrated that miR-367 was obviously upregulated in human NSCLC cancer tissues compared with that in the corresponding non-cancer tissues, and that the NSCLC patients with a high miR-367 expression had a significantly poorer prognosis than those with a low expression. Pearson's correlation analysis also provided evidence that a negative association existed between the expression of miR-367 and the FBXW7 protein in NSCLC patients. Furthermore, increasing evidence indicates that FBXW7 is significantly associated with the prognosis of cancer patients $(25,30-32)$. These findings suggest that the overexpression of miR-367 correlates with the poor prognosis of NSCLC patients, possibly due to the repression of the function of the FBXW7 protein.

Research has found that FBXW7-deficient mice died in utero of vascular abnormalities at embryonic days 10.5-11.5 (33). Additionally, although p53-deficient mice do not develop tumors, knockdown of FBXW7 verifies the tumor profile in p53-deficient mice and that they develop lung cancer (34). Moreover, suppression and mutation of FBXW7 have been reported to be involved in various types of malignancies (35-37). A number of studies have indicated that the loss or mutation of FBXW7 may exert a vital role in the development and progression of lung carcinogenesis. FBXW7 functions as a tumor regulator by controlling the expression of oncoproteins such as cyclin E, MYC, TOP2A, MCL1, and P53 which are all correlated with tumor development and progression $(12,13)$. These data, together with the correlation of FBXW7 between the prognosis of patients with tumors, suggest that FBXW7 functions as a tumor suppressor in human tumorigenesis. In the present study, we found that the expression of FBXW7 was significantly downregulated in the NSCLC clinical samples compared with the control and the expression levels of FBXW7 in cancer tissues were negatively associated with miR-367. As is well known, miRNAs modulate the expression of their target genes at the post-transcriptional level (38). They prevent their translation by directly binding to the corresponding complementary sequences of their target mRNAs, thereby downregulating protein expression. Many studies indicate that abnormal expression of miRNAs is associated with various human diseases, including malignancies (39-42). For instance, Lei et al identified the oncogenic function of miR-92b in NSCLC through targeting reversioninducing cysteine-rich protein with Kazal motifs (RECK). Zhang et al reported that miR-150 promoted the proliferation of NSCLC cells by targeting p53 (43). In contrast, miR-638 and miR-625 act as tumor suppressors via targeting sex determining region Y (SRY)-box 2 (SOX2), respectively (44). However, whether FBXW7 is post-transcriptionally regulated by miR-367 remains unclear. Therefore, we aimed to identify whether miR-367 was involved in the regulation of FBXW7. Notably, via using miRNA prediction programs, we found that a fragment of the FBXW7 3'-UTR contained the putative miR-367 binding site. To further confirm the prediction, miR-367 mimics were co-transfected with a luciferase reporter construct containing wild-type FBXW7 3'-UTR. Our results indicated that transfection with miR-367 mimics downregulated luciferase activity compared with transfection with NC. Furthermore, the inverse experiments further confirmed the above results, suggesting that miR-367 is a potential regulator of FBXW7. Therefore, we focused on miR-367 that may have significant effects on the progression and development of NSCLC. $\chi^{2}$ tests showed a significant statistical correlation between tumor size, tumor stage, metastasis and miR-367 expression. Based on the above correlation, we performed a series of functional experiments to test the effect of miR-367 on NSCLC cells. Our results demonstrated that overexpression of miR-367 promoted proliferation, invasion and inhibited apoptosis in various types of NSCLC cell lines. We first demonstrated that overexpression of miR-367 was correlated with a poorer prognosis of NSCLC patients. Because of the function of FBXW7 in cancers, we developed a series of rescue experiments to further study whether the functional effect of miR-367 on NSCLC cell lines was exerted via targeting FBXW7. Our findings confirmed our hypothesis that proliferation, invasion and apoptosis were reversed to some extent when we silenced FBXW7 in cells with low expression of miR-367 compared to the control. Additionally, we also confirmed that high miR-367 expression and low FBXW7 expression could serve as independent prognostic factors, respectively.

In conclusion, we identified and characterized the miR-367/ FBXW7 axis, which is involved in proliferation, apoptosis and invasion of NSCLC cells. Based on our findings, miR-367 and FBXW7 may act as a therapeutic target for the treatment of human NSCLC, especially cancers with high invasive potential. Additionally, miR-367 and FBXW7 expression could respectively serve as independent prognostic factors.

\section{Acknowledgements}

This study was supported in part by the National Natural Science Foundation of China (81572263), the Jiangsu Province Natural Science Foundation (BK20151584) and the Jiangsu Top Expert Program in Six Professions (WSW-028).

\section{References}

1. Villaruz LC, Kalyan A, Zarour H and Socinski MA: Immunotherapy in lung cancer. Transl Lung Cancer Res 3: 2-14, 2014.

2. Ricciuti B, Mecca C, Crinò L, Baglivo S, Cenci M and Metro G: Non-coding RNAs in lung cancer. Oncoscience 1: 674-705, 2014.

3. Landi L and Cappuzzo F: Management of NSCLC: Focus on crizotinib. Expert Opin Pharmacother 15: 2587-2597, 2014.

4. Gridelli C, Peters S, Sgambato A, Casaluce F, Adjei AA and Ciardiello F: ALK inhibitors in the treatment of advanced NSCLC. Cancer Treat Rev 40: 300-306, 2014.

5. Uramoto $\mathrm{H}$ and Tanaka F: Recurrence after surgery in patients with NSCLC. Transl Lung Cancer Res 3: 242-249, 2014.

6. Vallières E: Oligometastatic NSCLC: The changing role of surgery. Transl Lung Cancer Res 3: 192-194, 2014. 
7. McElnay P and Lim E: Adjuvant or neoadjuvant chemotherapy for NSCLC. J Thorac Dis 6 (Suppl 2): S224-S227, 2014.

8. Shcherba M, Liang Y, Fernandes D, Perez-Soler R and Cheng H: Cell cycle inhibitors for the treatment of NSCLC. Expert Opin Pharmacother 15: 991-1004, 2014.

9. Gentzler RD, Yentz SE, Johnson ML, Rademaker AW and Patel JD: The changing landscape of phase II/III metastatic NSCLC clinical trials and the importance of biomarker selection criteria. Cancer 120: 3853-3858, 2014

10. Gómez AM, Jarabo Sarceda JR, García-Asenjo JA, Fernandez C, Hernandez S, Sanz J, Fernandez E, Calatayud J, Torres A and Hernando F: Relationship of immunohistochemical biomarker expression and lymph node involvement in patients undergoing surgical treatment of NSCLC with long-term follow-up. Tumour Biol 35: 4551-4559, 2014

11. Grim JE: Fbxw7 hotspot mutations and human colon cancer: Mechanistic insights from new mouse models. Gut 63: 707-709, 2014.

12. Tu K, Zheng X, Zan X, Han S, Yao Y and Liu Q: Evaluation of Fbxw7 expression and its correlation with the expression of c-Myc, cyclin E and p53 in human hepatocellular carcinoma. Hepatol Res 42: 904-910, 2012

13. Guo Z, Zhou Y, Evers BM and Wang Q: Rictor regulates FBXW7-dependent c-Myc and cyclin E degradation in colorectal cancer cells. Biochem Biophys Res Commun 418: 426-432, 2012

14. Izumi N, Helker C, Ehling M, Behrens A, Herzog W and Adams RH: Fbxw7 controls angiogenesis by regulating endothelial Notch activity. PLoS One 7: e41116, 2012

15. Babaei-Jadidi R, Li N, Saadeddin A, Spencer-Dene B, Jandke A, Muhammad B, Ibrahim EE, Muraleedharan R, Abuzinadah M, Davis $\mathrm{H}$, et al: FBXW7 influences murine intestinal homeostasis and cancer, targeting Notch, Jun, and DEK for degradation. J Exp Med 208: 295-312, 2011.

16. Ren H, Koo J, Guan B, Yue P, Deng X, Chen M, Khuri FR and Sun SY: The E3 ubiquitin ligases $\beta$-TrCP and FBXW7 cooperatively mediates GSK3-dependent Mcl-1 degradation induced by the Akt inhibitor API-1, resulting in apoptosis. Mol Cancer 12: $146,2013$.

17. Ren H, Zhao L, Li Y, Yue P, Deng X, Owonikoko TK, Chen M, Khuri FR and Sun SY: The PI3 kinase inhibitor NVP-BKM120 induces GSK3/FBXW7-dependent Mcl-1 degradation, contributing to induction of apoptosis and enhancement of TRAIL-induced apoptosis. Cancer Lett 338: 229-238, 2013.

18. Li N, Lorenzi F, Kalakouti E, Normatova M, Babaei-Jadidi R, Tomlinson I and Nateri AS: FBXW7-mutated colorectal cancer cells exhibit aberrant expression of phosphorylated-p53 at Serine-15. Oncotarget 6: 9240-9256, 2015.

19. Zhou C, Shen L, Mao L, Wang B, Li Y and Yu H: miR-92a is upregulated in cervical cancer and promotes cell proliferation and invasion by targeting FBXW7. Biochem Biophys Res Commun 458: 63-69, 2015.

20. Calcagno DQ, Freitas VM, Leal MF, de Souza CR, Demachki S Montenegro R, Assumpcão PP, Khayat AS, Smith MA, dos Santos AK, et al: MYC, FBXW7 and TP53 copy number variation and expression in gastric cancer. BMC Gastroenterol 13 141,2013

21. Ibusuki M, Yamamoto Y, Shinriki S, Ando Y and Iwase H: Reduced expression of ubiquitin ligase FBXW7 mRNA is associated with poor prognosis in breast cancer patients. Cancer Sci 102: 439-445, 2011

22. Tan Y, Sangfelt O and Spruck C: The Fbxw7/hCdc4 tumor suppressor in human cancer. Cancer Lett 271: 1-12, 2008.

23. Kuiper RP, Vreede L, Venkatachalam R, Ricketts C, Kamping E, Verwiel E, Govaerts L, Debiec-Rychter M, Lerut E, van Erp F, et al: The tumor suppressor gene FBXW7 is disrupted by a constitutional $\mathrm{t}(3 ; 4)(\mathrm{q} 21 ; \mathrm{q} 31)$ in a patient with renal cell cancer. Cancer Genet Cytogenet 195: 105-111, 2009.

24. Park MJ, Taki T, Oda M, Watanabe T, Yumura-Yagi K, Kobayashi R, Suzuki N, Hara J, Horibe K and Hayashi Y: FBXW7 and NOTCH1 mutations in childhood T cell acute lymphoblastic leukaemia and $\mathrm{T}$ cell non-Hodgkin lymphoma. $\mathrm{Br}$ J Haematol 145: 198-206, 2009.

25. Morra F, Luise C, Merolla F, Poser I, Visconti R, Ilardi G, Paladino S, Inuzuka H, Guggino G, Monaco R, et al: FBXW7 and USP7 regulate CCDC6 turnover during the cell cycle and affect cancer drugs susceptibility in NSCLC. Oncotarget 6 : $12697-12709,2015$.
26. Qin C, Huang RY and Wang ZX: Potential role of miR-100 in cancer diagnosis, prognosis, and therapy. Tumour Biol 36 : 1403-1409, 2015.

27. Bennett PE, Bemis L, Norris DA and Shellman YG: miR in melanoma development: miRNAs and acquired hallmarks of cancer in melanoma. Physiol Genomics 45: 1049-1059, 2013.

28. Yokobori T, Yokoyama Y, Mogi A, Endoh H, Altan B, Kosaka T, Yamaki E, Yajima T, Tomizawa K, Azuma Y, et al: FBXW7 mediates chemotherapeutic sensitivity and prognosis in NSCLCs. Mol Cancer Res 12: 32-37, 2014.

29. Zhang Z, Zhang Y, Sun XX, Ma X and Chen ZN: microRNA-146a inhibits cancer metastasis by downregulating VEGF through dual pathways in hepatocellular carcinoma. Mol Cancer 14: 5 , 2015.

30. Natarajan V, Bandapalli OR, Rajkumar T, Sagar TG and Karunakaran N: NOTCH1 and FBXW7 mutations favor better outcome in pediatric South Indian T-cell acute lymphoblastic leukemia. J Pediatr Hematol Oncol 37: e23-e30, 2015.

31. Naganawa Y, Ishiguro H, Kuwabara Y, Kimura M, Mitsui A, Katada T, Tanaka T, Shiozaki M, Fujii Y and Takeyama H: Decreased expression of FBXW7 is correlated with poor prognosis in patients with esophageal squamous cell carcinoma. Exp Ther Med 1: 841-846, 2010.

32. Milne AN, Leguit R, Corver WE, Morsink FH, Polak M, de Leng WW, Carvalho R and Offerhaus GJ: Loss of CDC4/FBXW7 in gastric carcinoma. Cell Oncol 32: 347-359, 2010.

33. Tsunematsu R, Nakayama K, Oike Y, Nishiyama M, Ishida N, Hatakeyama S, Bessho Y, Kageyama R, Suda T and Nakayama KI: Mouse Fbw7/Sel-10/Cdc4 is required for notch degradation during vascular development. J Biol Chem 279: 9417-9423, 2004.

34. Mao JH, Perez-Losada J, Wu D, Delrosario R, Tsunematsu R, Nakayama KI, Brown K, Bryson S and Balmain A: Fbxw7/Cdc4 is a p53-dependent, haploinsufficient tumour suppressor gene. Nature 432: 775-779, 2004

35. Yang H, Lu X, Liu Z, Chen L, Xu Y, Wang Y, Wei G and Chen Y: FBXW7 suppresses epithelial-mesenchymal transition, stemness and metastatic potential of cholangiocarcinoma cells. Oncotarget 6: 6310-6325, 2015.

36. Sato M, Rodriguez-Barrueco R, Yu J, Do C, Silva JM and Gautier J: MYC is a critical target of FBXW7. Oncotarget 6: 3292-3305, 2015.

37. Li Z, Sun Y, Chen X, Squires J, Nowroozizadeh B, Liang C and Huang J: p53 mutation directs AURKA overexpression via miR-25 and FBXW7 in prostatic small cell neuroendocrine carcinoma. Mol Cancer Res 13: 584-591, 2015.

38. Pfeffer SR, Yang CH and Pfeffer LM: The role of miR-21 in Cancer. Drug Dev Res 76: 270-277, 2015.

39. Yang Y, Xie YJ, Xu Q, Chen JX, Shan NC and Zhang Y: Down-regulation of miR-1246 in cervical cancer tissues and its clinical significance. Gynecol Oncol 138: 683-688, 2015.

40. Ye H, Pang L, Wu Q, Zhu Y, Guo C, Deng Y and Zheng X: A critical role of mir-199a in the cell biological behaviors of colorectal cancer. Diagn Pathol 10: 65, 2015.

41. Han S, Yang S, Cai Z, Pan D, Li Z, Huang Z, Zhang P, Zhu H, Lei L and Wang W: Anti-Warburg effect of rosmarinic acid via miR-155 in gastric cancer cells. Drug Des Devel Ther 9: 2695-2703, 2015.

42. Liang J, Li X, Li Y, Wei J, Daniels G, Zhong X, Wang J, Sfanos K, Melamed J, Zhao J, et al: LEF1 targeting EMT in prostate cancer invasion is mediated by miR-181a. Am J Cancer Res 5: 1124-1132, 2015.

43. Zhang N, Wei $X$ and Xu L: miR-150 promotes the proliferation of lung cancer cells by targeting P53. FEBS Lett 587: 2346-2351, 2013.

44. Wang Z, Qiao Q, Chen M, Li X, Wang Z, Liu C and Xie Z: miR-625 down-regulation promotes proliferation and invasion in esophageal cancer by targeting Sox2. FEBS Lett 588: 915-921, 2014. 